\title{
BRIEF COMMUNICATION
}

\section{Cortisol Response to a Psychological Stressor and/or Nicotine}

\author{
OVIDE F. POMERLEAU' ${ }^{1}$ AND CYNTHIA S. POMERLEAU \\ Behavioral Medicine Program, Department of Psychiatry \\ University of Michigan School of Medicine, Ann Arbor, MI 48105
}

Received 23 October 1989

\begin{abstract}
POMERLEAU, O. F. AND C. S. POMERLEAU. Cortisol response to a psychological stressor and/or nicotine. PHARMACOL BIOCHEM BEHAV 36(1) 211-213, 1990. - The effects of a psychological stressor and nicotine upon corticosteroid release were investigated using a full factorial, repeated-measures design in eight moderate smokers. Sessions involved the presentation of either competitive mental arithmetic or reading aloud and either smoking a usual cigarette or sham smoking. Self-reported anxiety increased after exposure to competitive mental arithmetic, confirming the stressfulness of the procedure. Cortisol levels increased significantly in response to psychological stress and showed a trend towards a significant elevation over time in response to nicotine self-administration. The two manipulations in combination produced additive effects upon plasma cortisol. These findings underscore the usefulness of the corticosteroid response as a marker of the impact of different procedures and suggest that it may provide an indicator for exploring the mechanisms by which nicotine-stress interactions are mediated. Systematic research that varies temporal and other parameters involving nicotine and various stressors will be needed to resolve inconsistencies in the literature on smoking and anxiety in the context of stress.
\end{abstract}

Anxiety Cigarettes Cortisol Corticosteroids Mental arithmetic Nicotine Smoking Stress

ACUTE intake of nicotine via cigarette smoking has been shown to cause the release of adrenocorticotropic hormone (ACTH), which in turn increases cortisol levels in circulation $(2,17,20)$. In rodents, nicotine has been shown to stimulate corticotropin releasing factor from the hypothalamus via a nicotinic cholinergic pathway $(7,18)$, and direct application of nicotine to the hypothalamus significantly increases levels of ACTH (9) and corticosterone (3). Numerous studies have demonstrated that stress also elevates corticosteroid levels in both infrahumans and humans [e.g., $(1,4,19)]$. In the rat, Brenner and colleagues (1) have shown that both basal release and stress-induced release of corticosteroids are mediated by a nicotinic cholinergic pathway. Recently, Morse reported that restraint stress and repeated acute nicotine dosing produce additive effects upon circulating corticosterone in rabbits (10). The combined effects of psychological stress and smoking on corticosteroid release in humans, however, have not been examined systematically.

The present study was undertaken to examine the effects of stress and smoking, both singly and in combination, upon cortisol release. In view of Morse's findings in rabbits (10), we hypothesized that acute nicotine administration and acute psychological stress would produce additive effects. Accordingly, a full factorial, repeated-measures design involving four conditions (one session for each of the four possible combinations of psychological stress and nicotine) was conducted in smokers. Because it has frequently been suggested that nicotine has anxiolytic effects and that smoking therefore increases under stressful or anxiety-producing conditions, subjective anxiety and change in plasma nicotine were also measured.

\section{METHOD}

Eight male smokers (smoking at least 20 cigarettes per day for at least 5 years), in otherwise good health and not on medications, participated in the study. Mean age was $29.3 \pm 3.0$ (s.e.m.) years. They had smoked for 12.1 \pm 2.7 years and reported smoking a mean of $26.3 \pm 2.1$ cigarettes per day. Mean nicotine delivery of usual cigarettes was $1.04 \pm 0.07 \mathrm{mg}$. Mean score on the Fagerstrom Tolerance Questionnaire was $7.1 \pm 0.6$, and mean plasma cotinine level was $288.0 \pm 64.4$, characterizing these subjects as moderately nicotine-dependent.

In an initial telephone interview, subjects were taught mental arithmetic (subtracting 13's from a given four-digit number) and told to practice before coming in for the first scheduled session. Each subject participated in four sessions at least two days apart,

\footnotetext{
${ }^{1}$ Requests for reprints should be addressed to O. F. Pomerleau, Behavioral Medicine Laboratory, University of Michigan Department of Psychiatry, Riverview Building, 900 Wall Street, Ann Arbor, MI 48105.
} 
presented in Latin square order, to control for sequence effects. Sessions involved either a psychological stressor (competitive mental arithmetic) or no stress (reading National Geographic aloud), followed by either nicotine intake (smoking a usual cigarette) or no nicotine intake (sham smoking an unlit cigarette). Subjects sat quietly before the stress/smoking sequence and read quietly for 35 minutes afterwards. The stressfulness of the mental arithmetic was augmented by competition provided by promise of a monetary bonus of $\$ 50$ and $\$ 25$ for the top two scorers (13).

Sessions were conducted in late morning. Upon arrival at the laboratory, the subject smoked one usual-brand cigarette; a heparinized catheter leading to an opaque infusion line was inserted into a forearm vein for sampling of plasma nicotine and cortisol. The session began thirty minutes later, allowing time for the cortisol effects of venipuncture to subside and ensuring a uniform, minimal nicotine deprivation interval. Mental arithmetic/reading aloud was scheduled for ten minutes shortly after the beginning of the session (minutes 8 to 18) and was followed by smoking/ sham-smoking for five minutes (minutes 20 to 25). Preceding and following mental arithmetic (minutes 7 and 19), and following smoking (minute 26), subjective state was queried using visual analog scales presented on an AMIGA console.

Blood samples were taken $7,19,26,38,50$, and 62 minutes into the session. Samples were collected in EDTA tubes and kept in ice prior to centrifuging and storage in a $-80^{\circ} \mathrm{C}$ freezer at the end of the session. Plasma nicotine was quantitated by HPLC (5); the average coefficient of variation $(\mathrm{CV}=$ standard deviation divided by the mean) for the assay is $6.5 \%$ for nicotine and $4.0 \%$ for cotinine, with a lower limit of detectability of $1 \mathrm{ng} / \mathrm{ml}$ for nicotine and $3 \mathrm{ng} / \mathrm{ml}$ for cotinine in plasma. Cortisol was quantitated by competitive protein binding assay; average interassay $\mathrm{CV}$ is $6.3 \%$ and intraassay is $5.9 \%$, with a $0.5 \mathrm{microgram} / \mathrm{dl}$ limit of detection. Statistical analyses involving repeated measures ANOVA and ANCOVA were carried out using BMDP 2V; onetailed tests were used since directional hypotheses were specified.

\section{RESULTS}

To determine the effects of experimental manipulations upon self-reported anxiety, a 3-way ANOVA [2 levels of stress: mental arithmetic, reading aloud; by 2 levels of smoking: smoking, sham-smoking; by 3 levels of time: minute 7 (prestress), minute 19 (poststress/presmoking), minute 26 (postsmoking)] was conducted. Self-reported anxiety increased and then returned to baseline levels on the competitive mental arithmetic days, but not on the reading aloud days [stress by time; $\mathrm{F}(2,6)=5.05, p=0.026$ ]; changes in anxiety observed on smoking days did not differ significantly from those observed on sham-smoking days [smoking by time; $\mathrm{F}(2,6)=$ $0.61, \mathrm{NS}$ ]. No significant stress by smoking by time interaction was detected for anxiety, $F(2,6)=0.42$, NS.

Mean presmoking plasma nicotine level for the reading aloud condition was $19.1 \pm 3.7 \mathrm{ng} / \mathrm{ml}$ and postsmoking level was $37.5 \pm$ 7.6; presmoking level in the competitive mental arithmetic condition was $22.3 \pm 4.7$ and postsmoking level was $36.8 \pm 4.8$ [ANCOVA comparing postsmoking plasma samples (at 26 minutes), using presmoking values (at 19 minutes) as a covariate, $\mathrm{F}(1,6)=$ 0.52 , NS].

As can be seen in Fig. 1a, cortisol levels showed a secular downward trend, as would be expected for late morning values. In order to illustrate more clearly the effects of the experimental manipulations, the cortisol levels for the control condition (reading aloud/sham-smoking) were subtracted from the comparable time point in each experimental condition, as shown in Fig. 1b. Because nicotine and psychological stress were presented out of phase with one another, a 3-way ANCOVA (2 levels of stress, 2 levels of nicotine, and 4 levels of time) was conducted on the time
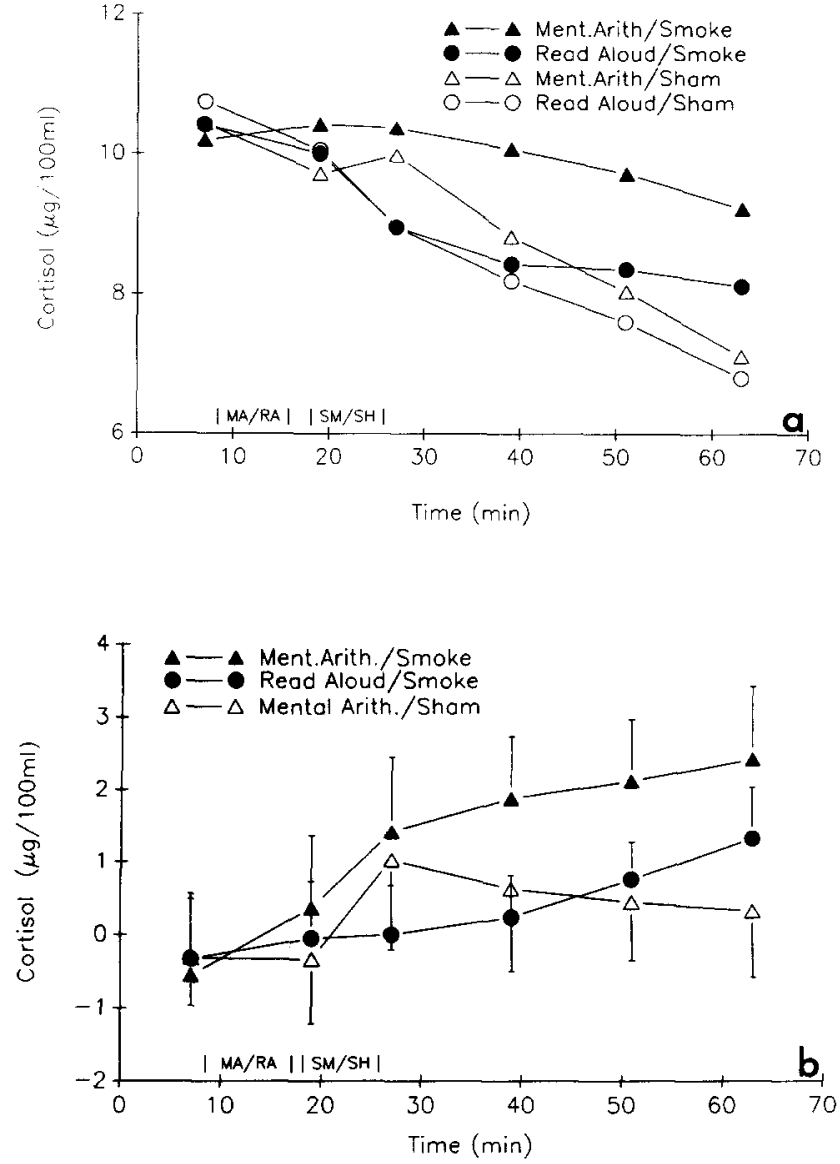

FIG. 1. (a) Plasma cortisol (mean) as a function of time for experimental conditions-mental arithmetic and smoking usual cigarette (MA/SM), reading aloud and smoking usual cigarette (RA/SM), mental arithmetic and sham smoking (MA/SH) - and control condition - reading aloud and sham smoking (RA/SH). (b) Plasma cortisol (mean \pm s.e.m.) as a function of time for experimental conditions. Cortisol levels for each time point in the control condition (RA/SH) were subtracted from the comparable timepoint in each experimental condition.

points that followed both manipulations (minutes $26,38,50$, and 62), using baseline (minute 7) values as a covariate. A significant overall stress effect, $\mathrm{F}(1,6)=5.71, p=0.027$, and a strong trend towards a significant overall effect for smoking, $F(1,6)=3.12$, $p=0.064$, were detected, along with a highly significant time effect, $\mathrm{F}(3,21)=19.9, p=0.000$, reflecting the secular downward trend in late morning cortisol levels. No significant stress by time effect was observed; a drug by time effect, $\mathrm{F}(3,21)=2.7, p=$ 0.036 , emerged, reflecting the persistent effects of nicotine upon cortisol in the postsmoking period. No overall interactive effects that is, stress by drug by time-were detected, $F(3,21)=0.58$, $\mathrm{NS}$, indicating that the effects of nicotine and stress over time were additive.

\section{DISCUSSION}

The change in perceived anxiety associated with competitive mental arithmetic confirms that the procedure was psychologically stressful (13). Anxiety changes for cigarette smoking were similar to those for sham-smoking, suggesting that reductions in selfreported anxiety observed at the end of the mental arithmetic interval were the result of termination of the stressor rather than 
nicotine self-administration. Though a number of studies have demonstrated nicotine's ability to alleviate anxiety $(6,14,16)$, the conditions under which this phenomenon reliably occurs are still not known (8).

The lack of a significant induction of nicotine intake in the stress condition is at odds with previous reports of stress-induced smoking [e.g., $(13,15)]$. Recent observations, however, suggest that the temporal relations between the stressor and smoking as well as the nature of the stressor (8) are critical. Systematic research that varies both the timing and intensity of nicotine intake and various stressors, and that includes measures of plasma nicotine, will be needed to determine the interrelationships between nicotine and anxiety in the context of stress.

When presented separately, psychological stress and nicotine intake elevated cortisol levels; the patterns of change, however, were different. Following a lag of several minutes after the presentation of the psychological stressor, cortisol levels increased and then decayed over the rest of the session. On the other hand, the rise in cortisol after nicotine intake was gradual, and the augmentation continued throughout the session, suggesting that the pharmacological effects of nicotine upon cortisol persisted at least 35 minutes after smoking. The combination of an acute stressor and nicotine produced additive effects upon cortisol.

These observations suggest that the corticosteroid response can serve as a marker of the impact of different procedures and may well provide a useful indicator for exploring the mechanisms by which nicotine-stress interactions are mediated (11). Systematic research examining the cortisol effects of both fixed dosing and self-administration of nicotine (12), and varying the temporal relations between stress and nicotine availability, may help to resolve some of the puzzling inconsistencies that characterize the literature on smoking and anxiety in the context of stress.

\section{ACKNOWLEDGEMENT}

Partial support for this research was provided by National Cancer Institute Grant CA 42730.

\section{REFERENCES}

1. Brenner, T.; Mizrachi, R.; Bodoff, M.; Weidenfeld, J. Evidence that nicotinic-acetylcholine receptors are involved in the modulation of basal and stress-induced adrenocortical responses. Exp. Neurol. 94:735-743; 1986.

2. Chiodera, L.; Cammelini, G.; Rossi, G.; Maffei, M. L.; Volpi, R.; Coiro, V. Effect of clonidine on ACTH and cortisol release induced by cigarette smoking in man. Horm. Metab. Res. 16:501-502; 1984.

3. Conte-Devolx, B.; Oliver, C.; Giraud, P.; Guillioz, P.; Castanas, E.; Lissistzky, J. C.; Boudouresque, F.; Millet, Y. Effect of nicotine on in vitro secretion of melanocorticotropic hormones in the rat. Life Sci. 28:1067-1073; 1981.

4. Frankenhauser, $M$. The rate of peripheral catecholamines in adaptation to understimulation and overstimulation. In: Serban, G., ed. Psychopathology of human adaptation. New York: Plenum; 1976: 173-191.

5. Hariharan, M.; Van Noord, T.; Greden, J. F. An HPLC method for the routine simultaneous determination of nicotine and cotinine in plasma. Clin. Chem. 34:724-729; 1988.

6. Heimstra, N. W. The effects of smoking on mood change. In: Dunn, W. L., ed. Smoking behavior: Motives and incentives. Washington, DC: V. H. Winston \& Sons; 1973:197-207.

7. Hillhouse, E. W.; Burden, I.; Jones, M. T. The effect of various putative neurotransmitters on the secretion of corticotrophin-releasing hormones from the rat hypothalamus in vitro-a model of the neurotransmitters involved. J. Endocrinol. 69:1-10; 1975.

8. Jarvik, M. E.; Caskey, N. H.; Rose, J. E.; Herskovic, J. E.; Sadeghpour, M. Anxiolytic effects of smoking associated with four stressors. Addict. Behav. 14:379-386; 1989.

9. Marty, M.; Erwin, V.; Cornell, K.; Zgombick, J. Effects of nicotine on Beta-endorphin, Alpha-MSH, and ACTH secretion by isolated perfused mouse brain and pituitary glands, in vitro. Pharmacol. Biochem. Behav. 22:317-325; 1985.

10. Morse, D. E. Neuroendocrine responses to nicotine and stress:
Enhancement of peripheral stress responses by the administration of nicotine. Psychopharmacology (Berlin) 98:539-543; 1989.

11. Pauly, J. R.; Ullman, E. A.; Collins, A. C. Adrenocortical hormone regulation of nicotine sensitivity in mice. Physiol. Behav. 44:109$116 ; 1988$

12. Perkins, K. A.; Epstein, L. H.; Jennings, R.; Stiller, R. The cardiovascular effects of nicotine during stress. Psychopharmacology (Berlin) 90:373-378; 1986.

13. Pomerleau, C. S.; Pomerleau, O. F. The effects of a psychological stressor on cigarette smoking and subsequent behavioral and physiological responses. Psychophysiology 24:278-285; 1987.

14. Pomerleau, O. F.; Turk, D. C.; Fertig, J. B. The effects of cigarette smoking on pain and anxiety. Addict. Behav. 9:265-271; 1984.

15. Rose, J. E.; Ananda, S.; Jarvik, M. E. Cigarette smoking during anxiety-provoking and monotonous tasks. Addict. Behav. 8:353-359; 1983.

16. Schachter, S. Pharmacological and psychological determinants of smoking. Ann. Intern. Med. 88:104-114; 1978.

17. Seyler, L. E.; Fertig, J. B.; Pomerleau, O. F.; Hunt, D.; Parker, K. The effects of smoking on ACTH and cortisol secretion. Life Sci. $34: 57-65 ; 1984$.

18. Weidenfeld, J.; Siegel, R.; Conforti, N.; Mizrachi, R.; Brenner, T. Effect of intracerebroventricular injections of nicotinic acetylcholine receptor antibodies on ACTH, corticosterone and prolactin secretion in the male rat. Brain Res. 265:152-156, 1983.

19. Williams, R. B.; Lane, J. D.; Kuhn, C. M.; Melosh, W.; White, A. D.; Schanberg, S. M. Type A behavior and elevated physiological and neuroendocrine responses to cognitive tasks. Science 218:483$485 ; 1982$.

20. Wilkins, J. N.; Carson, H. E.; van Vunakis, H.; Hill, M. A.; Gritz, E.; Jarvik, M. E. Nicotine from cigarette smoking increases circulating levels of cortisol, growth hormone, and prolactin in male chronic smokers. Psychopharmacology (Berlin) 78:305-308; 1982. 